\title{
Los eventos running en la localidad de Bahía Blanca (Argentina). Una aproximación a su impacto turístico y económico
}

\section{Running events in the city of Bahía Blanca (Argentina): An approximation to its tourism and economic impacts}

Viviana S. Leonardi ${ }^{1}$ \& Darío A. Miraglia².

Resumen: El impacto de eventos deportivos ha sido motivo de numerosas investigaciones. Actualmente el impulso de dichos eventos puede considerarse como una estrategia de desarrollo local. En la Argentina el turismo de eventos deportivos es una modalidad que moviliza miles de personas en torno a la gran cantidad y diversidad de eventos que se realizan tanto a nivel internacional, nacional como regional. Una práctica deportiva que está creciendo a gran velocidad en los últimos años es el running. En relación al turismo, la ciudad de Bahía Blanca, que es un centro de orden intermedio y se ubica en el sudoeste de la Provincia de Buenos, está posicionada como un lugar "de paso" y de distribución hacia destinos turísticos cercanos. El objetivo del presente trabajo es analizar el impacto turístico y económico de los eventos running en la localidad de Bahía Blanca. Dicho estudio se realiza utilizando un enfoque mixto de investigación, a partir de metodologías cualitativas y cuantitativas. Los principales resultados muestran que, si bien Bahía Blanca posee una gran variedad de atributos para el desarrollo del running como actividad turística, el amplio calendario de competencias existentes en la localidad no resulta ser muy convocante, ya que el mayor porcentaje de participantes proviene de localidades cercanas o son de Bahía Blanca. Por ello, el trabajo presenta propuestas para mejorar y diversificar la oferta de eventos running en la ciudad, como elemento dinamizador de la actividad turística.

Palabras clave: Turismo deportivo, eventos running, impacto turístico y económico.
Abstract: The impact of sporting events has been a deeply researched subject in the literature. At present, the promotion of these events can be considered as a local development strategy. In Argentina, sporting events tourism is an activity that attracts many people in relation to the great number and diversity of events that take place at international, national and regional levels. In recent years, running is a sport practice that has been growing quickly. In terms of tourism, Bahía Blanca, which is a city located in the southwest of the Province of Buenos Aires, is positioned as a "gateway" to tourist destinations nearby. The aim of this paper is to analyze the tourism and economic impacts of running events in Bahía Blanca. The study uses a mixed research approach, based on qualitative and quantitative methodologies. The main results show that, although Bahía Blanca has a great variety of attributes for the development of running as a tourist activity, the broad calendar of existing competitions in the city does not seem to be very attractive, since the greater percentage of participants comes from nearby locations or lives in Bahía Blanca. Therefore, this work presents proposals to improve and diversify the running events offer in the city, as an important element to boost the tourist activity.

Key Words: Sports tourism, running events, tourism and economic impact.

(Presentado: 30 de agosto de 2019. Aceptado: 5 de noviembre de 2019).

\footnotetext{
1 Licenciada y Magíster en Economía, docente e investigadora, Universidad Nacional del Sur- IIESS, UNS- CONICET. E-mail: viviana.leonardiQuns.edu.ar, Bahía Blanca, Argentina.

2 Licenciado en Turismo, Universidad Nacional del Sur. Graduado Departamento de Geografía y Turismo. E-mail: dam2806đgmail.com, Bahía Blanca, Argentina.
} 


\section{INTRODUCCIÓN}

La relación entre deporte y turismo ha sido estrecha desde sus comienzos y su principal antecedente son los Juegos Olímpicos celebrados en Atenas en 1896. Sin embargo, no fue hasta finales del siglo XX que dicha relación comenzó a ser abordada con la importancia que poseía y a partir de allí, una gran cantidad de autores y las principales instituciones a nivel mundial de cada actividad trataron el binomio deporte y turismo. Los hechos que marcaron un antes y un después fueron en primer lugar, el acuerdo de cooperación entre el Comité Olímpico Internacional (COI) y la Organización Mundial del Turismo realizado en 1999; y, en segundo lugar, la I Conferencia Mundial sobre Deporte y Turismo, celebrada en 2001 en Barcelona (Latiesa y Paniza, 2006).

Los cambios sociales, económicos y la revalorización por parte de las personas por mejorar su calidad de vida en las últimas décadas, han sido los principales propulsores de ambas actividades, el tiempo libre y el tiempo de ocio, se integran hoy en la vida cotidiana, no como una actividad aislada, sino como una parte más de la vida diaria. La característica más significativa de este tiempo de ocio, es la libertad de elección y su principal objetivo es el disfrute personal y social. Por otra parte, la presencia del deporte, entendido como elemento potenciador de la calidad de vida, está asociado al concepto de recreación. Por ello, el deporte entendido como actividad física recreativa, está determinado y definido por una serie de características, como son la diversión y placer como finalidad principal, y otras como la salud, relación social y relax (Nogueras, 2010).

El resultado de la relación deporte-turismo y su desarroIlo, es el denominado turismo deportivo, el cual Latiesa y Paniza (2006: 136), definen como "toda aquella actividad relacionada con el deporte que se lleva a cabo fuera del lugar habitual de residencia, ya sea en instalaciones artificiales o en el medio natural para realizar una actividad física u observar espectáculos deportivos, pudiendo existir o no fines competitivos". González Molina, A. (2008), afirma que el mismo responde a la búsqueda de nuevos retos personales, y tienen una clara proyección social y objetivos saludables. Además su principal motivación es la realización de prácticas físico-deportivas muy concretas, que requieren técnica, un buen estado físico, infraestructuras de organización y reglas especificadas claramente.

Brasileiro, Rebollo y Medina (2008:78) afirman que "el binomio turismo y deporte constituye un mercado de gran rentabilidad social y económica", destacando así que el turismo deportivo genera importantes impactos positivos en la localidad en la cual se llevan a cabo. El análisis del impacto de los eventos deportivos sobre la economía fue analizado por diferentes autores, los cuales consideran que la celebración de dichos eventos puede ser un elemento de desarrollo socioeconómico y una opción estratégica para mejorar la competitividad de las ciudades (Burgan y Mules, 1992; Tyrrell y Johnston, 2001; Daniels 2003; entre otros).

En Argentina, el turismo deportivo es una modalidad que moviliza miles de personas en torno a la gran cantidad y diversidad de eventos que se realizan a nivel internacional, nacional, y regional. Entre los eventos más destacados es posible mencionar el Moto GP como gran evento de interés mundial, el automovilismo en todas sus categorías, el fútbol, básquet, running, ciclismo, natación y triatlones, entre otras.

En la ciudad de Bahía Blanca (Argentina), donde el turismo de negocios es la principal modalidad, debido a su localización geográfica, a la infraestructura que posee, la cercanía al Polo Petroquímico y al Puerto de Bahía Blanca, los eventos deportivos han comenzado a ser de gran importancia para la ciudad, debido a la cantidad de visitantes que moviliza, con Olimpo participando del campeonato de $1^{\circ}$ División del Fútbol Argentino, Weber Bahía participando de la Liga Nacional de Básquet y la Liga de las Américas y el Torneo Estival de Midget.

En relación al running, en la actualidad existe un amplio calendario de competencias pedestres que se desarrollan en la localidad y que representan una alternativa al turismo deportivo. El objetivo del presente trabajo es analizar la potencialidad turística de eventos runners en Bahía Blanca (Argentina) y su impacto económico a partir del estudio de la demanda y la oferta de competencias pedestres con la finalidad de realizar propuestas para mejorar la atractividad de esta práctica deportiva. La importancia de esta investigación radica en servir como disparador para conocer las características de la oferta y demanda de dichos eventos a los fines de delinear propuestas que posibiliten a los hacedores de política deportiva local, mejorar la potencialidad turística de los mismos.

\section{ASPECTOS METODOLÓGICOS}

El análisis empírico emplea una perspectiva cuantitativa de investigación basada en el análisis e interpretación de infor- 
mación tanto secundaria como primaria. En primer lugar, para el estudio de la demanda y oferta de eventos running en Bahía Blanca, se utiliza información secundaria suministrada por páginas web especializadas en la temática, como BahíaCorre. $\mathrm{com}^{3}$ (página web local) y Frieni.com ${ }^{4}$ (página web nacional). En segundo lugar, para analizar el perfil de las personas que practican running, conocer cuáles son sus preferencias a la hora de participar en un evento fuera de su ciudad y el gasto que realizan, se utilizan datos primarios provenientes de una encuesta llevada a cabo en los meses de Julio y Agosto de 2017. Dichas encuestas se realizaron a personas que practican running en Bahía Blanca. La encuesta fue diseñada a partir de un formulario de Google. Es importante destacar que la utilización de esta herramienta posee limitaciones como medio de implementación de encuestas, como por ejemplo, deja de lado a un segmento importante de la población ya que solo permite llegar a aquellas personas que poseen internet y el control de la selección de los informantes se dificulta. Sin embargo, la misma posee varias ventajas como realizar encuestas de una manera rápida y sencilla, y la ausencia de intermediarios entre el cuestionario y el entrevistado posibilita mayor objetividad y menores costos en el relevamiento.

El número total de personas encuestadas fue de 103. El tamaño de la muestra se determinó sobre una población de 1000 personas que practican este deporte en forma activa en la localidad. Se consideran activos a aquellos que participan al menos de dos eventos en el año. Este número fue determinado sobre la información suministrada por el entrenador de uno de los grupos más importantes de la ciudad. Considerando que para poblaciones finitas $(\mathrm{N}<100.000)$ la fórmula generalmente empleada es $n=Z 2 p q /(e 2+Z 2 p q / N)$, el número de encuestas realizadas permite trabajar con un nivel de confianza del $90 \%$. El tamaño de muestra es representativo de la población. A partir de la información obtenida se desarrolla un análisis exploratorio-descriptivo a los fines de estudiar el impacto turístico y económico de eventos running en la localidad de Bahía Blanca, para luego realizar propuestas con el propósito de mejorar la potencialidad turística de los mismos.

\section{MARCO DE REFERENCIA}

\section{El turismo de eventos deportivos}

Es posible afirmar que los eventos deportivos representan un gran volumen del total de eventos que se organizan a lo largo de un año en el mundo. Teniendo en cuenta el interés social, económico y mediático del deporte, son muchos los eventos deportivos, de mayor o menor envergadura que se celebran.

Añó Sanz (2000: 271) define a los eventos deportivos como "aquella actividad deportiva que cuenta con un alto nivel de repercusión social traducido en una fuerte presencia en los medios de comunicación y que genera por sí misma ingresos económicos". El autor hace referencia a los eventos de gran dimensión, y que atrae a un enorme volumen de visitantes. Según este autor, una actividad deportiva para transformarse en un evento deportivo, debe poseer las siguientes características: (i) repercusión social, (ii) nivel amplio de asistencia de público, (iii) presencia de los medios de comunicación, (iv) audiencia televisiva, (v) patrocinadores y (vi) ingresos propios.

Desde la perspectiva organizativa los eventos deportivos pueden ser organizados por una tipología variada de organizaciones, tales como organismos nacionales deportivos, clubes, asociaciones, federaciones, empresas, instituciones educativas, etc. (Graham, 2001, en Cerezuela 2003). No existe una tipología específica a la hora de organizar un evento deportivo, existen una gran cantidad de ejemplos que así lo demuestran, desde una Copa Mundial de Fútbol, hasta las Maratones más importantes del mundo, como la de Nueva York, o Berlín y las media maratones realizadas a lo largo y a lo ancho del mundo. La tendencia en la Argentina, presenta una tipología de organización en la cual participan organismos públicos, como también privados, con una importante presencia de estos últimos a través de la publicidad, aportando recursos económicos para su realización.

Respecto al turismo de eventos deportivos, Delpy (2003) lo define como "aquellas actividades o competiciones deportivas capaces de atraer un número considerable de visitantes con el objeto de participar o asistir como espectadores" (citado en Revuelta, 2006:2). Esta modalidad de turismo deportivo ha adquirido una gran relevancia, constituyéndose en la actualidad, en una gran oportunidad para los destinos que buscan aumentar sus atractivos, tanto por el número de turistas que atraen como por su impacto económico (Getz, 2003 citado en Alles, 2014).

Numerosos autores destacan que esta modalidad turística genera importantes impactos positivos en la localidad en la cual se realizan (Burgan y Mules, 1992; Tyrrell y Johnston, 2001; Daniels 2003; Daniels 2004; entre otros). Sánchez-Fernández, P., Barajas, Á., \& Alén González, M. E. (2013) subrayan que entre los aspectos beneficiosos para los territorios están el incremento

\footnotetext{
${ }^{3}$ www.bahiacorre.com.ar es la página web de Bahía Corre, organización fiscalizadora de eventos deportivos sin fines de lucro.

${ }^{4}$ www.frieni.com es una página web que gestiona y publicita eventos deportivos con o sin fines de lucro.
} 
de los ingresos, la construcción de instalaciones y la mejora de la imagen de la localidad. Por su parte Jago y Dwyer (2006), plantean que la realización de un evento genera una inyección inicial de dinero que tiene un impacto directo en la economía local y otro secundario. El impacto directo procede del gasto realizado por los visitantes en los distintos sectores de la economía. El impacto secundario se relaciona con el efecto multiplicador del dinero inicial inyectado en la economía (Sánchez-Fernández, P., Barajas, Á., \& Alén González, M. E. ,2013). Asimismo Crompton (2006) define el impacto económico como el flujo de gasto atribuido a los visitantes y convertido en ingresos para la comunidad anfitriona.

En la literatura, pueden encontrarse trabajos que han analizado el impacto de mega eventos (Brunet, 1992; Baade y Matheson, 2004; Baade, Baumann y Matheson, 2010; entre otros), de eventos de tamaño medio y de menor escala (WiIson, 2006, Késenne et al., 2011; Barajas y Sánchez, 2011; entre otros). También se destacan trabajos que analizan las dificultades del turismo de eventos deportivos (Getz, 1997 y 1998) y estudian cuestiones vinculadas a la planificación, el desarrollo y el marketing en este tipo de turismo (Getz, 2003).

Los eventos deportivos, al igual que otro tipo de eventos como culturales, artísticos o deportivos, pueden constituirse en una gran oportunidad para el desarrollo turístico de ciudades, regiones o países en la medida que incentivan el desarrollo socioeconómico local, contribuyendo a la generación de empleos, así como a la creación y mejora de las infraestructuras (Alles, 2014:62).

Todo lo anterior pone de manifiesto que el desarrollo de eventos deportivos puede convertirse en una herramienta de desarrollo local. Como afirman Henderson et. Al. (2010:60), ciertos eventos tienen la capacidad de atraer un gran número de turistas, así como el gasto que realizan, de ahí el interés de los agentes públicos en atraerlos por su contribución actual y potencial en el desarrollo del turismo (Alles, 2014: 60).

\section{Nuevas tendencias: El running}

Es importante destacar que correr como actividad física es una disciplina específica del atletismo y la misma presenta diferentes versiones. Estas van desde las pruebas más famosas de velocidad de 100 y 200 metros llanos, a los emblemáticos 42 kilómetros del maratón como expresión más representativa de la carrera de fondo o resistencia. Durante muchos años y hasta aproximadamente 1960, los atletas que corrían, lo hacían con la intención de entrenarse en la disciplina que se especializaban o deporte que practicaban como complemento de su preparación física.

Bill Bowerman fue la persona que impulsó el cambio en el running como actividad masiva. Bowerman fue un reconocido entrenador de atletismo en la década de 1950, en Oregón, Estados Unidos y formó una gran cantidad de deportistas. Sin embargo, a comienzos de 1960 su visión del atletismo como actividad cambió bruscamente al observar que en Nueva Zelanda, existían grupos de personas que conformaban grupos de "trote" con la finalidad de mejorar su condición física, mejorar su salud o simplemente por gusto y disfrute. Luego, trasladó la idea a Eugene, su localidad natal, junto con el médico Harris con la finalidad de evaluar la salud de los participantes a medida que concurrían a las salidas de trote. Por otra parte, observó que la convocatoria poco a poco se hizo masiva en su localidad y comenzó a extenderse a otras ciudades de los Estados Unidos. En 1967 Bowerman y Harris publicaron un libro titulado "Jogging: A physical Fitness program for all ages” que vendió más de un millón de ejemplares y ayudó a difundir en forma masiva lo que ellos llamaban trote. En él se destacaban que trotar, "es un tipo de ejercicio sencillo que no requiere habilidades altamente desarrolladas. Su gran atractivo es su sencillez misma, casi cualquiera puede practicarlo y en cualquier parte" (Atletas, 2014).

Otro de los grandes impulsores de la actividad física para mejorar la calidad de vida de las personas fue Kenneth Cooper, médico de las fuerzas armadas de los Estados Unidos, que publicó en 1968 el libro "Aerobics" (Crummy, 2016). Luego de lo realizado tanto por Bowerman y Harris, como por Cooper, apareció primero en EE.UU.y, posteriormente, en otros países del mundo la moda del jogging/running, que es la forma más popular de practicar un entrenamiento aeróbico de resistencia. A principio de los '70 la práctica del "jogging o footing” siguió creciendo y en paralelo se dio un suceso deportivo que le aportó aceleración a su crecimiento. En 1972 se disputaron los Juegos Olímpicos de Múnich y en la prueba principal de maratón, ganó el atleta americano Frank Shorter y arribó en 4to lugar otro atleta americano, Kenneth Moore, ya que la última vez que había ganado esa prueba un estadounidense, había sido en 1908. Este suceso dio un impulso extraordinario a la incipiente movida del running transformando el atletismo en una práctica popular en los Estados Unidos (Atletas, 2014).

Años posteriores, en 1977 James Fixx publicó "El libro completo del corredor" que se transformó inmediatamente en un éxito, en donde describía las bondades de la práctica de running: aumento de oxígeno en la sangre; bombeo de la sangre por el corazón con menos presión; incremento 
de aire para los pulmones; desarrollo y fortaleza muscular; desarrollo de más y mejor resistencia, etc. Sin embargo, Fixx no sólo hizo mención a las mejoras en la salud y en las funciones del cuerpo humano, sino que considero al running como una "diversión" más en sus vidas, correr por diversión y no por salud, o para obtener un buen cuerpo, o para ganar. En esto consistió el secreto de su éxito editorial y mediante el cual, aportaron masividad al fenómeno y contribuyeron a que personas distantes al deporte, se acerquen en busca de mejorar problemáticas de salud y vitalidad (EI Nuevo Diario, Nicaragua, 2009).

El running ya era una señal distintiva de la sociedad norteamericana y comenzaba a extenderse por el resto del mundo. Las marcas deportivas aprovecharon esta movida y acompañaron el proceso. Repentinamente comenzaron a aparecer empresas y nuevas tecnologías que apoyaron e incentivaron al masivo grupo de runners que se sumaba día a día. Había nacido una nueva disciplina, el running como expresión popular y abierta a las masas era un hecho.

Por otra parte, en las últimas décadas, el crecimiento del running, como factor de atracción ha sido considerablemente alto. Existen innumerables ejemplos que así lo demuestran, como la Maratón de Buenos Aires, creada en 1984 y que se ha convertido en la más convocante de América Latina en 2016, con más de 10.000 participantes provenientes de diversas partes del mundo. También en Buenos Aires, se lleva a cabo la Media Maratón, en donde han participado más de 20.000 personas en las últimas ediciones y se ha convertido en la competencia más importante de la Argentina.

El running puede considerarse como un atractivo turístico. Siguiendo a Kotler et al. (2007:185), un atractivo turístico puede hacer referencia tanto a las características físicas del destino como a los eventos que se desarrollan en él, siendo el objetivo fundamental de estos últimos convocar ciudadanos, nuevos residentes, visitantes, empresarios e inversionistas.

Por último, el aspecto más importante del running como atractivo turístico, es el número de días que permanecen los corredores en el destino donde se desarrolla la competencia, ya que pernoctan en destino 2 ó 3 días y consumen diversos servicios durante su estadía. Es por el tipo de viaje realizado que beneficia en gran medida no solo a la organización del evento, sino también al área de servicios en general y por consiguiente a la localidad (Miraglia, 2017).

\section{El caso de la ciudad de Bahía Blanca}

\section{El área de estudio}

A los $38^{\circ} 44^{\prime}$ de Latitud Sud y $62^{\circ} 16^{\prime}$ de Longitud Oeste del meridiano de Greenwich, se emplaza la ciudad de Bahía Blanca, ubicada en el frente costero del sudoeste de la provincia de Buenos Aires (Argentina) y es la cabecera del partido homónimo (Figura 1). El mismo está conformado por las ciudades de Bahía Blanca, Ingeniero White, General Daniel Cerri y Cabildo, y limita con los partidos de Tornquist, Coronel Rosales, Villarino y Coronel Pringles (Municipalidad de Bahía Blanca, Área Turismo).

La ciudad cuenta con una población de 301.572 habitantes (Censo 2010), lo que la convierte en el decimoséptimo conglomerado urbano del país. A nivel regional, es el centro urbano más importante debido a la actividad portuaria e industrial y a su gran valor cultural, comercial, financiero, académico, deportivo y sanitario. A su vez, gracias a su importante infraestructura vial, ferroviaria y aeroportuaria, funciona como un nodo de transporte y comunicaciones de relevancia nacional, convirtiéndola en centro de escala.

La localidad es un centro de paso para turistas que se dirigen a otras partes del país, y también el centro de distribución dentro del Sudoeste de la provincia de Buenos Aires, no sólo por las características propias de Bahía Blanca, sino porque se encuentra localizado en un lugar estratégico en cuanto a la cercanía de importantes localidades turísticas como Sierra de la Ventana, Monte Hermoso, Pehuen Có, entre otras, las cuales se encuentran aproximadamente a 100 km de Bahía Blanca. Debido a la pluralidad de actividades culturales, científicas y recreacionales, entre otras, la actividad turística se ha incrementado notablemente (Gambarota y Leonardi, 2016), lo cual queda reflejado en el crecimiento observado en la ocupación hotelera en el período 2009-2017, siendo éste del $14 \%{ }^{5}$. Es de destacar que la ciudad cuenta con una variada oferta turística, respecto al equipamiento hotelero. Según la Asociación de Hoteles, Restaurantes, Bares, Confiterías y Afines de Bahía Blanca y región del Sudoeste, cuenta con más de 40 establecimientos de alojamientos, con un total aproximado de 2.200 plazas. La oferta comprende hoteles de 1, 2, 3, y 4 estrellas, además de motel, hostel, apart hotel y alojamiento residencial. Hay que destacar que la gran mayoría de dichos establecimientos se encuentran ubicados en la zona de micro y macrocentro.

${ }^{5}$ Instituto Nacional de Estadísticas y Censos (INDEC) y Ministerio de Turismo. Encuesta de Ocupación Hotelera. https://www.indec.gob.ar. Septiembre de 2009-2017.

Julio - Diciembre 2019 | 117 
Figura № 1. Localización del área de estudio.

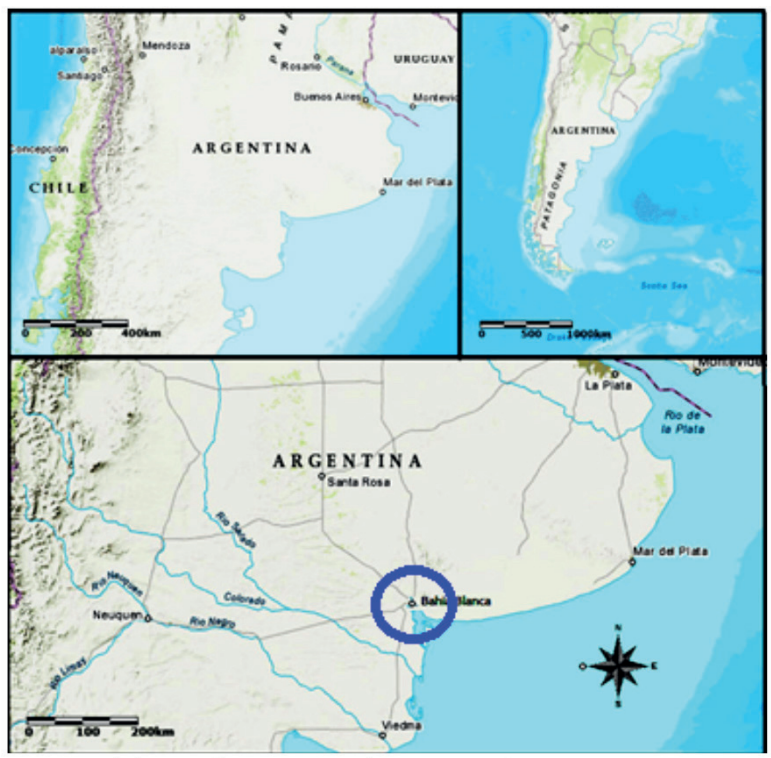

Fuente: Elaboración propia en base a ArcGIS.

\section{Análisis de oferta y demanda de eventos de running}

\section{Análisis de la oferta}

La oferta de competencias de running en la ciudad de Bahía Blanca es diversa aunque concentrada en la oferta de carreras de corta distancia, es decir, carreras a recorrer distancias de 10 kilómetros o menos. Solo una competencia de las 17 que conforman el calendario 2016, es considerada una competencia de media distancia ya que deben recorrerse 21 kilómetros. La misma se organiza desde el año 2011 y es la única media maratón certificada de la ciudad y la región (media maratón de Barlovento). El calendario de competencias en el año 2016 se presenta en la Tabla 1.

Tabla No 1. Calendario de Competencias en el 2016.

\begin{tabular}{|c|c|c|c|}
\hline Mes & Día & Competencia & Distancia \\
\hline Enero & 5 & Circuito de Reyes & $8.5 \mathrm{k}$ \\
\hline Febrero & - & - & - \\
\hline \multirow[t]{2}{*}{ Marzo } & 20 & IV Edición Integrar & \\
\hline & & Yo corro por la inclusión & $8.5 \mathrm{k}$ y $3 \mathrm{k}$ caminata \\
\hline \multirow[t]{3}{*}{ Abril } & 3 & Sanidad Corre & 8k y 3k caminata \\
\hline & 17 & Bahía 10k & $10 \mathrm{k}$ \\
\hline & 24 & Nativa & $5 k$ \\
\hline \multirow[t]{2}{*}{ Mayo } & 8 & Cross UNS & $6 \mathrm{k}$ \\
\hline & 25 & Barlovento & $21 \mathrm{k}, 14 \mathrm{k}$ y $7 \mathrm{k}$ \\
\hline Junio & - & - & - \\
\hline Julio & - & - & - \\
\hline \multirow[t]{2}{*}{ Agosto } & 7 & 100 Años Club Independiente & $10 \mathrm{k}$ \\
\hline & 14 & Sindicato Argentino de Televisión & $7 \mathrm{k}$ \\
\hline Septiembre & 11 & Carrera Aniversario Asociación Bahiense de Atletismo & 10k y $3 \mathrm{k}$ caminata \\
\hline \multirow[t]{3}{*}{ Octubre } & 16 & Corrida del Farmacéutico & $10 \mathrm{k}$ \\
\hline & 23 & Correcaminata Club Pacifico & $10 k$ y $4 k$ \\
\hline & 30 & Empleados de Comercio & $8 \mathrm{k}$ \\
\hline \multirow[t]{2}{*}{ Noviembre } & 20 & UNS Corre & $8 \mathrm{k}$ y $3.5 \mathrm{~K}$ caminata \\
\hline & 26 & Creer Sí & $5 \mathrm{k}$ \\
\hline \multirow[t]{2}{*}{ Diciembre } & 4 & Color Light Run & $10 k$ y $5 k$ \\
\hline & 8 & 10K STMBB & 10k y 3k caminata \\
\hline
\end{tabular}

Fuente: Elaboración propia sobre datos obtenidos de la página BahíaCorre.com. 
Las competencias más importantes son el "Circuito de Reyes", "Yo corro por la inclusión" y "Bahía 10 K", no sólo por el poder de convocatoria que poseen, sino también porque son los eventos en los cuales participa un mayor número de actores, tanto del sector público como privado, además que los tres eventos son declarados año tras año de interés municipal por el Honorable Concejo Deliberante de la Municipalidad de Bahía Blanca.

La primera carrera mencionada es el circuito más importante de la ciudad y en la actualidad ya se han realizado 60 ediciones. Es organizada por la Asociación Atlética Alumni y la Municipalidad de Bahía Blanca y se caracteriza por ser uno de los eventos atléticos más

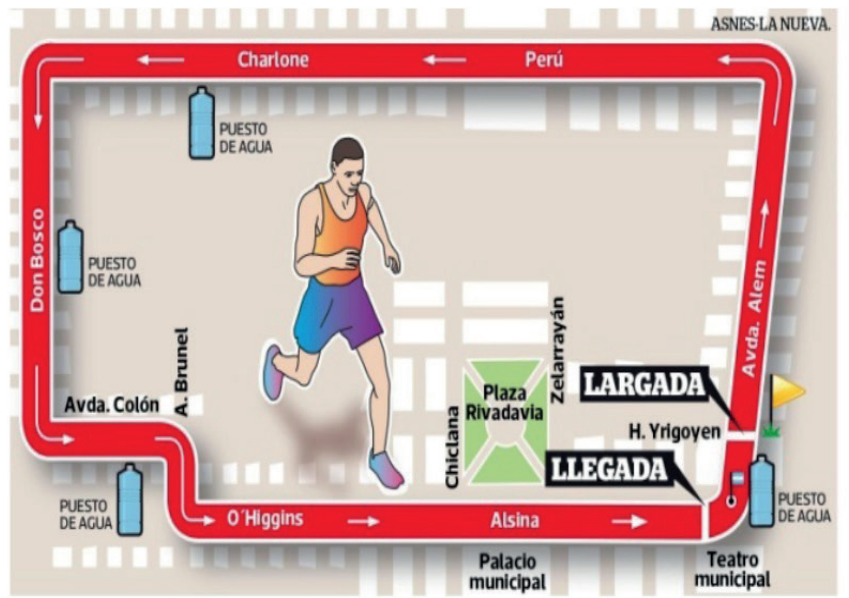

Figura 2. Recorrido Circuito de Reyes. antiguos de la Argentina. En la Figura 2 se observa el histórico y característico circuito de la competencia, donde se recorren $8.5 \mathrm{~km}$. El costo de inscripción a la misma en el año 2017 fue de \$150. La segunda competencia en orden de importancia, denominada yo corro por la inclusión, es la organizada por la ONG Integrar, que lucha por los derechos de las personas con síndrome de down, la misma se realiza desde el año 2013 en un circuito único creado por la Municipalidad de Bahía Blanca, que se observa en la Figura 3, dicho circuito se adapta para que su distancia sea de $8.5 \mathrm{~km}$ y además se realiza una caminata de $3 \mathrm{~km}$. Tanto para la competencia de $8.5 \mathrm{~km}$ como la camita tuvieron un costo fue de $\$ 200$ por persona en el año 2017.

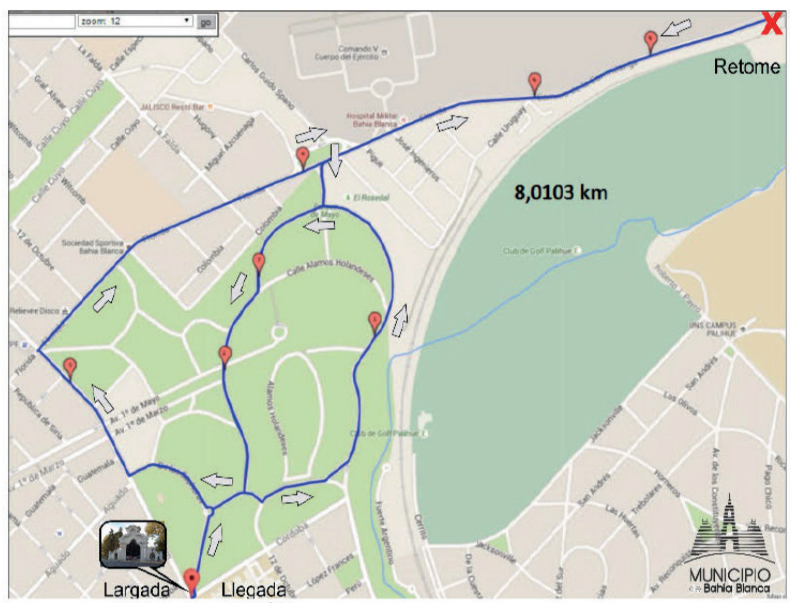

Figura 3. Circuito Único 8 k Bahía Blanca.

Fuente: Municipalidad de Bahía Blanca, 2016.

La competencia 10 k Bahía Blanca, es organizada por la Subsecretaria de Deportes de la Municipalidad de Bahía Blanca en conmemoración de la fecha de fundación de la ciudad, la misma se realiza desde el año 2009. El circuito se observa en la Figura 4 y es la competencia más convocante de $10 \mathrm{k}$ de la ciudad con más de 600 participantes en la edición del año 2017 y en el 2016 más de 700 participantes. Su costo en el año 2017 ascendió a $\$ 150$ por persona.

\section{Figura N 4. 10 k Bahía Blanca.}

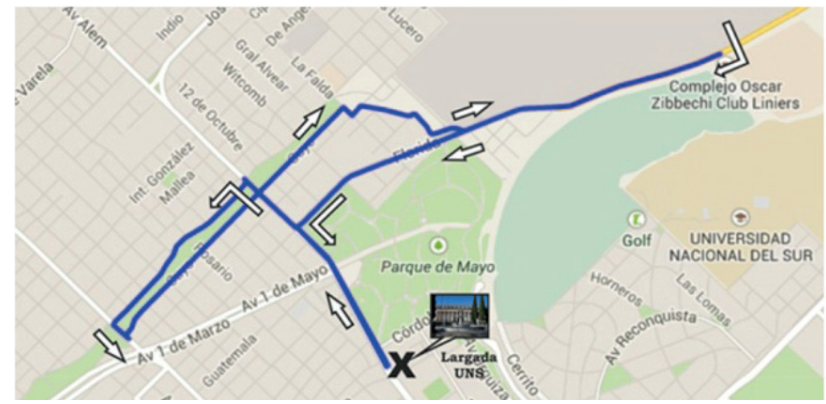

Fuente: Municipalidad de Bahía Blanca, 2016. 
En relación al costo de las tres competencias señaladas, si se compara este valor con otras competencias en localidades de la Provincia de Buenos Aires, se observa que es considerablemente menor. Por ejemplo, para competir en una competencia de similar distancia (10K) en Sierra de la ventana ${ }^{6}$ (Buenos Aires), el precio de inscripción fue de $\$ 550$, en Necochea y Mar del Plata, de $\$ 300$ y para participar de la Media Maratón de Mar del Plata, fue de \$350, siendo un $75 \%$ más costosa que la Media Maratón de Barlovento en Bahía Blanca (\$200). En Bahía Blanca, las carreras con el mayor costo de inscripción (\$250), fueron "La Corrida del Farmacéutico" y "Maratón del Sindicato de Empleados de Comercio". Un aspecto relevante de las mismas, es que fueron dos de las competencias que menor cantidad de corredores captó.

Por último, es necesario destacar, que la Secretaría de Deportes de la Municipalidad de Bahía Blanca, ha creado el denominado Circuito Único, con el objeto de llevar a cabo las competencias pedestres sin entorpecer en gran medi- da el tránsito vehicular de la ciudad, lo cual también permite disminuir los costos para la organización del evento y para el municipio. Por tal motivo, 11 de las 17 competencias que se desarrollaron en el 2016, se realizaron en el Circuito Único cumpliendo el objetivo planteado anteriormente. Sin embargo, su implementación llevó a que las competencias sean similares y no muy atractivas para las personas de la ciudad y la región, provocando una disminución en el interés de los corredores por participar en dichos eventos.

\section{La demanda histórica y real de eventos running}

El análisis de la demanda histórica de eventos running se realizó para el período 2010-2017, sobre datos obtenidos de las tres principales competencias organizadas en la ciudad de Bahía Blanca, el "Circuito de Reyes", "10 K Bahía” y "Yo corro por la inclusión". La Tabla 2 presenta la evolución en el número de participantes de las tres carreras antes mencionadas.

\section{Tabla $N^{\circ}$ 2. Número de participantes en tres de las principales carreras realizadas en la ciudad de Bahía Blanca}

\begin{tabular}{ccccc}
\hline Año & Circuito de Reyes & 10 K Bahía & Yo corro por la inclusión & Total \\
\hline 2010 & 1093 & 1327 & - & 2420 \\
2011 & 1402 & 2154 & - & 3556 \\
2012 & 1996 & 695 & - & 2691 \\
2013 & 2012 & 1123 & 891 & 4026 \\
2014 & 2676 & 577 & 940 & 4193 \\
2015 & 2960 & 638 & 948 & 4546 \\
2016 & 3079 & 732 & 1030 & 4841 \\
2017 & 3200 & 638 & 1104 & 4942 \\
Total & 18418 & 7884 & 4913 & 31215 \\
\hline
\end{tabular}

Fuente: www.bahiacorre.com.ar

La Tabla 2 permite observar que en el período analizado se produjo un importante crecimiento en el número total de inscriptos en las carreras estudiadas. La tasa anual acumulada (T.A.A.) arrojó un valor de 0,44 lo cual indica que año a año la cantidad de inscriptos en las tres principales carreras creció un $44 \%$.
La Figura 5 revela que la cantidad de participantes crece a lo largo del tiempo, mientras que la Figura 6 presenta la evolución en el número de participantes para las tres principales carreras. Claramente se visualiza que la más importante y la que ha crecido más en el tiempo es el "Circuito de reyes".

\footnotetext{
${ }^{6}$ Sierra de la Ventana es una localidad turística ubicada a $125 \mathrm{~km}$ al noreste de la ciudad de Bahía Blanca.

${ }^{7}$ La TAA se calculó como (VF/VI) 1/n-1 -1, donde VF es el valor final de la variable y VI el valor inicial.
} 
Figura $N^{\circ} 5$. Análisis de serie de tiempo. Evolución en la cantidad de participantes en las tres principales carreras.

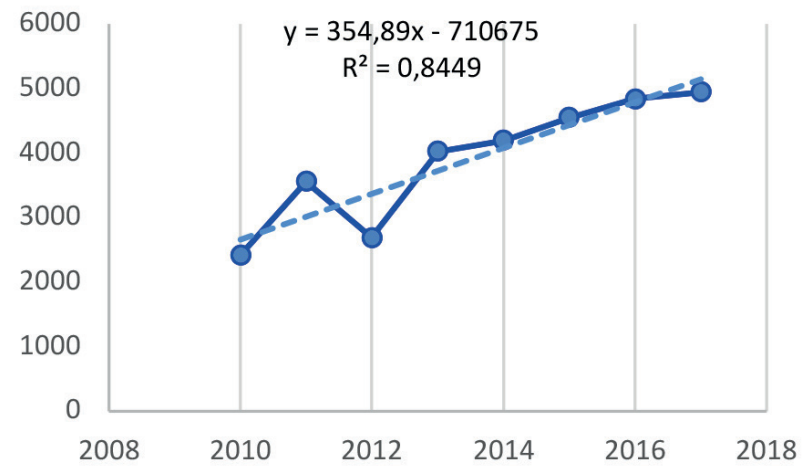

Fuente: Elaboración propia sobre datos de Bahía Corre (www.bahiacorre. com.ar

Figura $N^{\circ}$ 6. Evolución en el número de participantes por carrera.

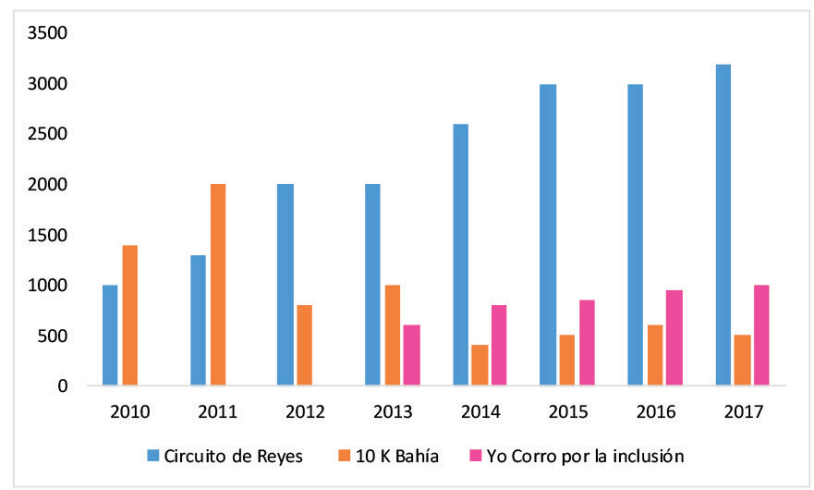

Fuente: Elaboración propia sobre datos de Bahía Corre (www.bahiacorre. com.ar)

Luego, se llevó a cabo el estudio de demanda real de los eventos running realizados en la ciudad de Bahía Blanca. Dicho análisis se formalizó a través de información secundaria y los datos recolectados, provienen de la organización fiscalizadora de eventos deportivos "Bahía corre". La información corresponde a ocho carreras del calendario de eventos running realizados en el año 2016.
Las características más destacadas de la demanda se presentan a continuación:

- El número resultante del total de participantes fue de 6.103 corredores distribuidos en las 8 competencias, siendo el "Circuito de reyes" el evento más atractivo.

- El $87 \%$ de los participantes, fue procedente de la localidad de Bahía Blanca, es decir 5.305 corredores, y el $13 \%$ restante fueron visitantes, sumando 798 participantes. La Figura 7 detalla la procedencia de los visitantes, que en su mayoría son de localidades de la Provincia de Buenos Aires, específicamente del sudoeste de la Provincia y Viedma. El $67 \%$ de los visitantes que recibió la ciudad de Bahía Blanca en los eventos analizados, provienen de localidades cercanas ubicadas como máximo, a 250 kilómetros de distancia. El restante $33 \%$ lo completan representantes de más de 30 localidades de la provincia de Buenos Aires en su mayoría, y también del resto del país.

- El "Circuito de reyes" fue la carrera más convocante del calendario de running de la ciudad, en donde participaron oficialmente 3.079 deportistas, con un elevado porcentaje de personas de Bahía Blanca (un $88 \%$ de la totalidad) que equivale a 2.699 personas.

- En algunas de las carreras, el número de participantes fue menor a los 200 corredores, con un resultado negativo no sólo para la organización, sino también para el calendario de running de la ciudad.

- Los resultados indican que los hombres, participan en mayor medida en las competencias de larga distancia, por ejemplo, en la única competencia de larga distancia que se desarrolló en la ciudad, "Media maratón de Barlovento" (21k), fue la competencia donde el porcentaje de hombres ( $82 \%)$, superó ampliamente a la de mujeres (18\%).

- Las competencias de menor distancia (5k y 7k), las mujeres igualaron, e incluso superaron el número de participantes masculinos.

- La mayoría de las organizaciones que realizaron las competencias a lo largo del 2016, establecieron un precio promedio de $\$ 150$. Las carreras con el mayor costo de inscripción fueron, la "Corrida del farmacéutico" y la organizada por el Sindicato de Empleados de Comercio, el mismo fue de $\$ 250$ pesos. Un aspecto relevante de las mismas, es que fueron dos de las competencias que menor cantidad de público captó. 
Figura $N^{\circ}$ 7. Procedencia de los participantes de las competencias del 2016.

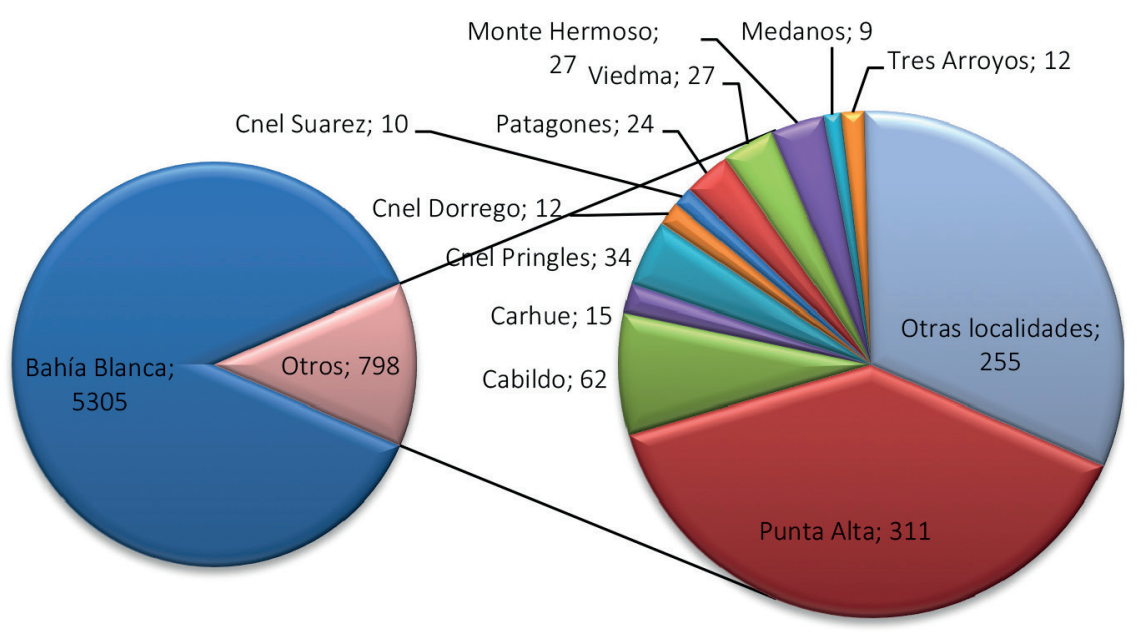

Fuente: Elaboración propia sobre datos de Bahía Corre (2016).

\section{Demanda potencial de eventos running}

En este apartado se busca conocer el perfil de las personas que practican running en Bahía Blanca, sus preferencias a la hora de participar en un evento running, determinar el gasto que realizan en promedio en cada uno de los eventos de los cuales participan y conocer su valoración de las propuestas que se presentan para mejorar la potencialidad turísticas de esta modalidad de turismo deportivo en la ciudad. Para el desarrollo, como ya se mencionó, se llevaron a cabo 103 encuestas a personas que practican running activamente, considerando corredor activo a aquellos que participan al menos de dos eventos en el año.

La información obtenida de la encuesta se presenta de la siguiente manera: (i) Perfil de las personas que practican running, (ii) Cantidad de días que permanece en el destino y gasto diario, y (iii) Aspectos valorados a la hora de seleccionar un evento en un destino y disponibilidad a pagar por un evento deportivo.

(i) Perfil de las personas que practican running:

Del análisis de los resultados de las encuestas, se observa que el $52 \%$ de los deportistas que participan de eventos son de género masculino, superando así al género femenino. Con respecto a la edad, se visualiza que el running es una actividad que abarca a todas las edades, incluidas a aquellos que tienen más de 60 años. Los rangos etarios que van de los 29 años a los 34 , de los 35 a 39 y de los 40 a 44, conforman más de la mitad de las personas encuestadas (Figura 8). Uno de los aspectos que más caracteriza a las personas que practican running es su formación académica, tal cómo se presenta en la Figura 9, aproximadamente el 75\% poseen estudios universitarios o terciarios.

Figura $N^{\circ}$ 8. Edad.
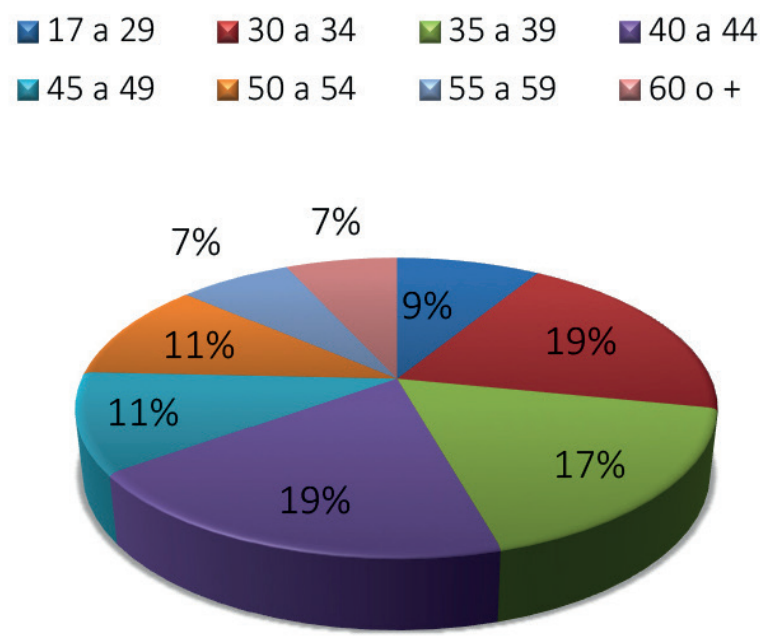

Fuente: Elaboración propia en base a encuesta 
Figura № 9. Máximo nivel de educación alcanzado.

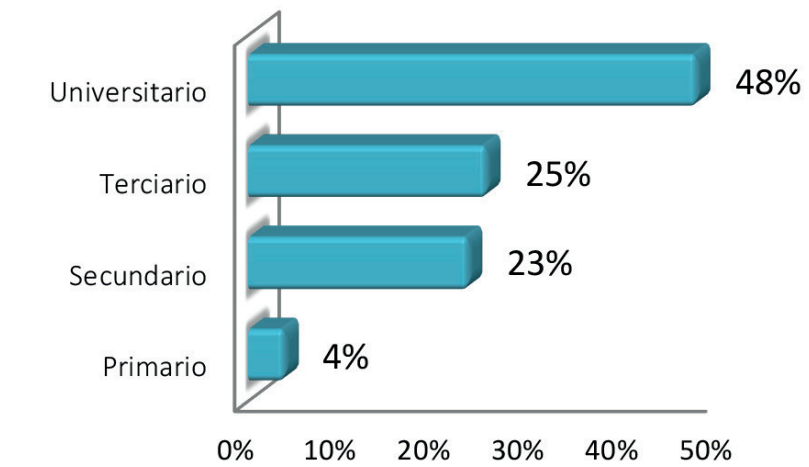

Fuente: Elaboración propia en base a encuesta.

(ii) Cantidad de días que permanece en el destino y gasto diario:

Parte de la preparación para los eventos, incluye los días de descanso que poseen los corredores en el destino donde se llevará a cabo la competencia, por tal motivo, consideran fundamental el arribo 2 ó 3 días previo a la competencia. En la Figura 10 puede observarse la respuesta a la pregunta: si participa en eventos en otras ciudades, ¿Cuantos días suele permanecer?

Como se demuestra en la Figura 10, de las 103 personas encuestadas el $48 \%$ suelen permanecer dos días en el destino, el $27 \%$ tres días o más y $16 \%$ solo lo hace un día. El menor porcentaje (9\%) solo permanece en el destino el día de la competencia, lo cual implica que no se desplaza una gran cantidad de kilómetros para participar de un evento. La Figura 11 presenta las respuestas a la pregunta, durante su estadía: ¿Cuánto gastó por día? Incluyendo alojamiento, traslados y comidas.

Figura $\mathrm{N}^{\circ} 10$. Cantidad de días que los corredores permanecen en el destino.

$\checkmark$ Sólo el día de la competencia $\square 1 \square 2 \square 30+$

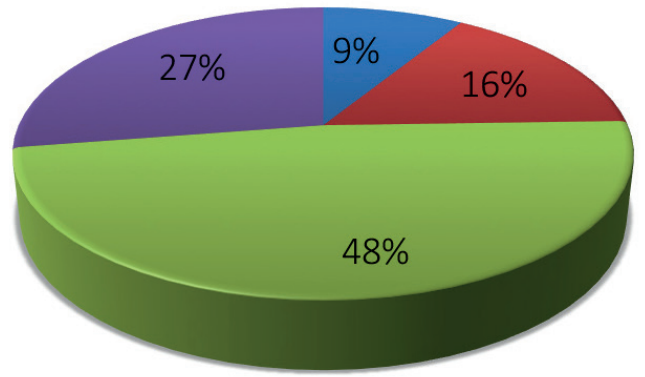

Fuente: Elaboración propia en base a encuesta.
Figura No 11. Gasto diario por día incluyendo alojamiento, traslado y comida.

Menos de $\$ 750$ घntre $\$ 750$ y \$1500 घ Más de \$1500

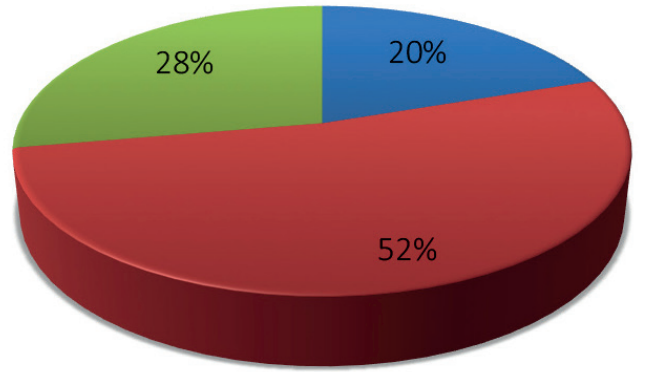

Fuente: Elaboración propia en base a encuesta.

En relación al gasto que realizan en el destino, la Figura 11 permite observar que el 52\% gastó entre $\$ 750$ y $\$ 1500$ por día, es decir, el $52 \%$ gastó en promedio $\$ 1.125$ por día, el $28 \%$ más de $\$ 1500$ y el menor porcentaje (20\%) gastó menos de $\$ 750$. Una demostración del gasto diario que realiza una persona que practica running al participar de un evento, es el tipo de alojamiento que utiliza los días que permanece en el destino. El $40 \%$ de las personas de las 98 que respondieron la pregunta: ¿En qué tipo de alojamiento se suele hospedar?, lo hacen en hoteles 3 estrellas o superior (Figura 12). Por otra parte, los departamentos son uno de los alojamientos más elegidos y cabe destacar, que en la opción "otros", un importante número de personas prefieren las cabañas como alojamiento.

Figura $N^{\circ} 12:$ Tipo de alojamiento que elige para hospedarse.

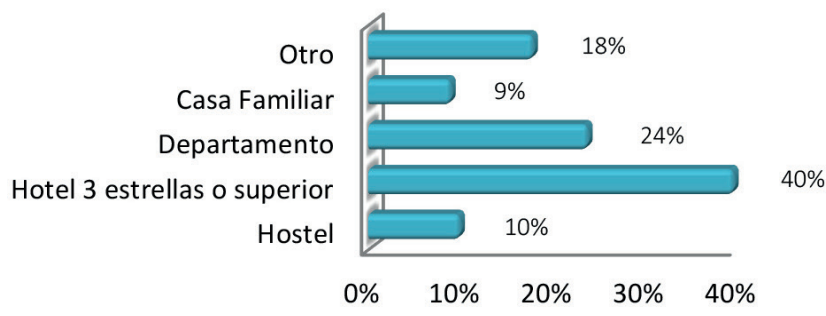

Fuente: Elaboración propia en base a encuesta. 
(iii) Aspectos valorados a la hora de seleccionar un evento en un destino y disponibilidad a pagar:

Mediante la encuesta se buscó conocer cuáles son los gustos y preferencias a la hora de elegir un destino para competir en un evento running. Para ello se preguntó qué valora del destino en el cual se realiza la carrera. El 51\% respondió que son los recursos naturales existentes en el destino el aspecto más valorado, fundamentalmente por el tipo de circuito que recorrerán en la competencia ya que las competencias de running aventura o cross running suelen diferenciarse en gran medida del resto.

Por otra parte, aquellas ciudades que son percibidas por las personas como importantes destinos turísticos obtuvo cerca de un $30 \%$ de las respuestas. Sin embargo, enmarcadas en la respuesta "otro/s", es necesario subrayar que existe una gran cantidad de variables que influyen a la hora de elegir el destino, pero que tienen relación con el evento en sí, como por ejemplo, la organización del mismo, el número de participantes, la distancia y circuito a recorrer y la importancia del evento, entre otras. Además es necesario resaltar que a las personas que practican running en grupo, les interesa asistir a eventos donde participen un gran número de integrantes del mismo grupo de entrenamiento. Por tal motivo, es de suma importancia a la hora de organizar un evento, convocar no sólo a los que participan individualmente, sino a los grupos de running, ya que son los que aportan una mayor cantidad de participantes.

La Figura 13 presenta el porcentaje de respuestas obtenido a la pregunta: ¿Qué valora del destino en el cual se realiza la carrera? Es necesario destacar que las personas que practican running, le dan una gran importancia a los circuitos de running aventura, ya que lo consideran uno de los principales factores de atracción porque representa un desafío personal variando según las condiciones de cada uno de los participantes.

Luego, se consultó acerca de cuánto han pagado para participar de un evento. La Figura 14 revela que cerca del 50\% han abonado más de $\$ 1000$, el $29 \%$ entre $\$ 500$ y $\$ 1000$ y solo el $24 \%$ pagó como máximo menos de $\$ 500$. Además, en un gran número de casos, han llegado a pagar U\$S700 por participar en "El Cruce Columbia", uno de los eventos más famosos e importantes de América del Sur. Sin embargo, para participar de un evento en Bahía Blanca, los encuestados mencionaron que el precio promedio que estarían dispuestos a pagar sería de $\$ 150$, siendo a su vez el valor mínimo a pagar en Bahía Blanca para participar de un evento.

\section{Figura $\mathrm{N}^{\circ} 13$. Aspectos valorados a la hora de seleccio- nar un evento en un destino.}

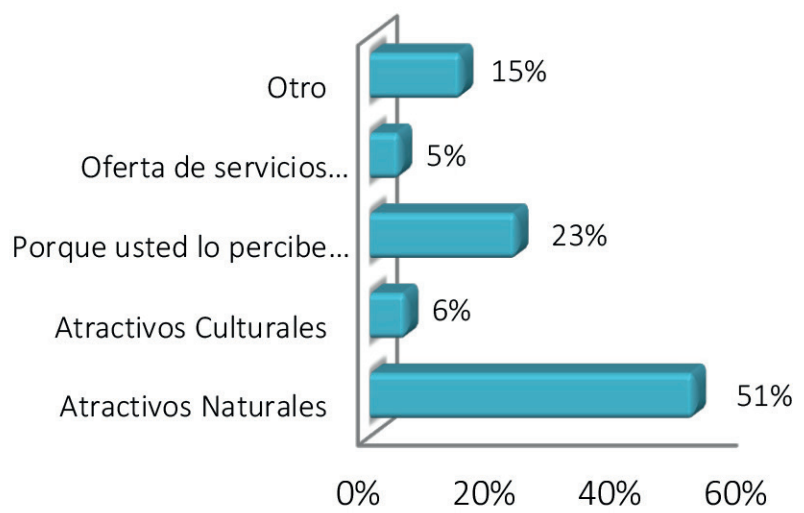

Fuente: Elaboración propia en base a encuesta.

\section{Figura $N^{\circ} 14$. Monto máximo pagado para participar de un evento.}

$\square$ Menos de $\$ 500 \square$ Entre $\$ 500$ y $\$ 1000 \square$ Más de $\$ 1000$

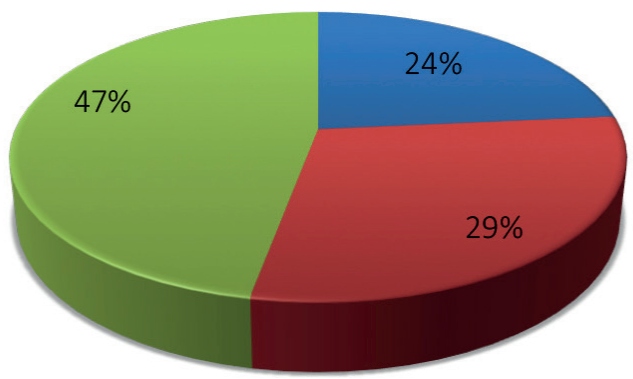

Fuente: Elaboración propia en base a encuesta.

\section{La incidencia de los eventos running en la economía local}

El análisis de la incidencia de eventos running en la economía local se realiza siguiendo lo planteado por Crompton (2006). Como ya se mencionó, dicho autor define el impacto económico como el flujo de gasto atribuido a los visitantes y convertido en ingresos para la comunidad anfitriona. 
Considerando la cantidad de turistas y visitantes que participaron de los eventos running realizados en Bahía blanca en el año 2016 (Figura 7) y teniendo en cuenta el gasto promedio que manifiestan realizar los corredores al inscribirse en un evento (Figura 11), se realiza la estimación de ingresos generados para la localidad. La estimación se formaliza considerando lo siguiente:

- Del total de participantes de eventos running en Bahía Blanca, solo el $13 \%$ resultaron ser visitantes, lo que arroja un total de 798 corredores (Figura 7).

- De los 798 corredores que participaron de eventos running el 53\%, es decir 423 corredores, provienen de localidades cercanas ubicadas a menos de $100 \mathrm{~km}$ de
Bahía Blanca, por lo cual se estable el supuesto que solo permanecen un día en la ciudad.

- Se considera que los restantes 375 corredores permanecen dos días en Bahía Blanca ya que provienen de localidades ubicadas a más de $100 \mathrm{~km}$ de la ciudad.

- La distribución de los gastos diarios en traslado, alojamiento y comida, efectuados por los participantes de los eventos es la siguiente: $52 \%$ gasta en promedio $\$ 1.125$, el $28 \%$ realiza un gasto promedio de $\$ 1.500$ y el $20 \%$ solo gasta $\$ 750$.

A partir de los datos presentados se realizan los siguientes cálculos presentados en la Tabla 3.

\section{Tabla $N^{\circ}$ 3. Gasto realizado por los participantes de eventos running que provienen de otras localidades.}

\begin{tabular}{|c|c|c|c|}
\hline $\begin{array}{l}\text { Cantidad de corredores que } \\
\text { permanecen un día en el destino } \\
\text { multiplicado por participación } \\
\text { en el gasto. }\end{array}$ & $\begin{array}{l}\text { Gasto promedio } \\
\text { por participante } \\
\text { año } 2016 \\
\text { (\$) }\end{array}$ & $\begin{array}{l}\text { Cantidad de días que } \\
\text { permanecen en } \\
\text { el destino }\end{array}$ & $\begin{array}{l}\text { Gasto expresado } \\
\text { en pesos argentinos } \\
\text { (\$año 2016) }\end{array}$ \\
\hline $423 * 0,52=220$ & $\$ 1.125$ & 1 & $\$ 247.500$ \\
\hline $423 * 0,28=119$ & $\$ 1.500$ & 1 & $\$ 178.500$ \\
\hline $423 * 0,20=85$ & $\$ 375$ & 1 & $\$ 31.875$ \\
\hline Subtotal & & & $\$ 457.875$ \\
\hline \multicolumn{4}{|l|}{$\begin{array}{l}\text { Cantidad de corredores que } \\
\text { permanecen dos días en el } \\
\text { destino multiplicado por } \\
\text { participación en el gasto. }\end{array}$} \\
\hline $375 * 0,52=195$ & $\$ 1.125$ & 2 & $\$ 438.750$ \\
\hline $375 * 0,28=105$ & $\$ 1.500$ & 2 & $\$ 315.000$ \\
\hline $375 * 0,20=75$ & $\$ 375$ & 2 & $\$ 56.250$ \\
\hline Subtotal & & & $\$ 810.000$ \\
\hline Total & & & $\$ 1.267 .875$ \\
\hline
\end{tabular}

Fuente: Elaboración propia en base al trabajo de campo

Observando la tabla 3 se destaca en primer lugar que los eventos realizados en la ciudad no resultan ser relevantes como factor de atracción de turistas o visitantes, y en segundo lugar, que el gasto realizado por dichos visitantes representa un ingreso de $\$ 1.267 .875$ para la economía local. A este gasto es necesario sumarle el pago que deben realizar los corredores para participar de las diferentes carreras en concepto de inscripciones. Como ya se mencio- nó el valor promedio de las carreras en Bahía Blanca en el año 2016 fue de $\$ 150$ y el número total de participantes en las ocho competencias analizadas de 6.103 corredores, por lo tanto, el ingreso generado en concepto de inscripciones fue de $\$ 915.450$.

En resumen, puede decirse que los eventos analizados en la localidad en el año 2016 aportaron un ingreso de 
$\$ 915.450$ en concepto de inscripciones y de $\$ 1.267 .875$ en gastos en alojamiento, traslado y comida. En cuanto al número de participantes inscriptos en los diferentes eventos, puede decirse que no resultaron ser altamente convocantes, por ello a continuación se presentan dos propuestas que podrían incrementar la potencialidad turística de los mismos.

Propuestas para mejorar y diversificar la oferta de eventos running en Bahía Blanca

En la encuesta realizada, se presentaron dos propuestas de eventos deportivos vinculados al running y ambas obtuvieron un importante porcentaje de aceptación. Las dos propuestas se realizaron en base a la necesidad de la ciudad de Bahía Blanca de diversificar la oferta de competencias pedestres que posee en la actualidad. Cómo se observó en el análisis de la oferta, aquellos eventos que se realizaron entre los meses de noviembre y marzo, tuvieron una mayor convocatoria, por tal motivo, se sugiere que las siguientes propuestas sean realizadas en estos meses.

Propuesta $\mathrm{N}^{\circ} 1$. Crear un evento que incluya una expo deportiva. Nombre del evento: "Feria runner Bahía 21k". La primera propuesta consiste en realizar una expo deportiva los días previos a la competencia y una carrera certificada de $21 \mathrm{k}$ (media maratón) conjuntamente con una de $8 \mathrm{k}$ participativa y una caminata de $3 \mathrm{k}$. El circuito de la competencia principal, será recorriendo los espacios más atractivos de la ciudad, cómo la avenida Alem, la avenida Colón, la calle Alsina, Sarmiento, Urquiza, Paseo de las Esculturas, Camino de la Carrindanga y Paseo de la Mujer, entre otros. En la "expo" habrá stands de marcas y locales deportivos, locales y nacionales, como así la exposición de las nuevas colecciones de indumentaria no sólo para la práctica de running, como también stands de profesionales de la salud especializados, como por ejemplo, en la mejora de las técnicas de los corredores y en patologías sufridas a causa de la práctica deportiva. Además, habrá disertaciones por parte de referentes del running, entre ellos, licenciados en educación física, nutricionistas, médicos deportólogos y maratonistas olímpicos. La distancia a recorrer se ha establecido en $21 \mathrm{k}$ (media maratón) dado que se ha observado que las personas suelen desplazarse una mayor cantidad de kilómetros, abonar una inscripción costosa y hospedarse más de un día, en aquellas competencias en las que se recorren $21 \mathrm{k}$ (media maratón), o distancias más extensas, por ejemplo, maratones, ultra maratones, etc.
Esta primer propuesta obtuvo un 95,2 \% de aprobación por parte de los encuestados.

Propuesta $\mathrm{N}^{\circ} 2$. Crear un circuito que incluya la ciudad, la sierra y el mar. Nombre del evento: "Circuito ciudad, mar y sierra”.

Los resultados arrojados por las encuestas realizadas han demostrado que un gran porcentaje de los corredores, prioriza los atractivos naturales que ofrecen los destinos, $y$ cómo Bahía Blanca es un destino urbano, es necesario combinar un evento con destinos de sol y playa y de montaña.

La siguiente propuesta consiste en una competencia pedestre de tres días que se desarrollará en tres lugares diferentes, un circuito urbano en Bahía Blanca, uno serrano en Sierra de la Ventana y por último un circuito playero en Monte Hermoso. Se utilizará a Bahía Blanca como ciudad principal del evento y de distribución hacia Monte Hermoso y Sierra de la Ventana. Las distancias que se recorrerán en cada localidad, serán de $10 \mathrm{~km}$. Habrá un ganador por competencia, pero se computarán los tiempos realizados en las tres competencias para determinar el ganador de la misma.

Esta segunda propuesta obtuvo un $99 \%$ de aprobación por parte de las personas encuestadas. La organización de las competencias requerirá un trabajo conjunto con el municipio de Bahía Blanca, Monte Hermoso, Tornquist y Coronel Suarez.

\section{CONCLUSIONES}

El turismo deportivo representa un fenómeno en constante desarrollo. El cambio que se ha dado en los últimos años en el ámbito social, respecto al interés por parte de las personas en mejorar su calidad de vida y su vinculación directa con la práctica deportiva ha impulsado a la actividad del running. Año a año el número de personas que realizan actividad física diariamente ha aumentado y el running es un claro ejemplo de ello. La demanda del running se caracteriza por ser completamente heterogénea, la misma es una actividad que la practican personas de todas las edades y ambos géneros, como también de diferentes sectores económicos, pero que tienen un factor en común, realizar una actividad física vinculada al disfrute y a mejorar su calidad de vida.

En referencia a Bahía Blanca, la ciudad posee una gran variedad de atributos para el desarrollo del running como 
actividad turística, la infraestructura y el equipamiento necesario para albergar importantes eventos, cómo la posibilidad de desarrollar atractivos circuitos urbanos. Además, presenta una ubicación estratégica en el sudoeste de la provincia de Buenos Aires y una importante oferta histórica, cultural y comercial. Sin embargo, la ciudad no cuenta con atractivos naturales, un factor importante para los participantes de los eventos runners al momento de seleccionar un destino, por tal motivo, destinos como Monte Hermoso y Sierra de la Ventana son importantes competidores en lo que respecta al running.

Asimismo, Bahía Blanca cuenta con un amplio calendario de competencias, siendo tres las que resultan más atractivas tanto para la población local como regional, el "Circuito de reyes", "10K Bahía" y "Yo corro por la inclusión". El análisis de la demanda histórica de estos tres eventos demuestra que se ha producido un importante crecimiento en el número de participantes en el período 2010-2017. Sin embargo, el mayor porcentaje de participantes son locales o provienen de localidades cercanas, lo que indica que podría mejorarse las condiciones de atractividad de dichos eventos, y por lo tanto, el ingreso que los mismos generan para la localidad.

Dos aspectos a mejorar están relacionados con la distancia que se recorre en cada circuito y con la definición del propio circuito. Se observó que recorren distancias que no superan los $10 \mathrm{~km}$, es un aspecto negativo al momento de analizar los eventos como atractivos turísticos, ya que mediante el trabajo de campo quedó demostrado que las personas se desplazan una mayor cantidad de kilómetros y destinan mayor cantidad de dinero a aquellas competencias de mediana o larga distancia, cómo lo son las media maratones o maratones. Otro aspecto negativo es la definición de un circuito único para el desarrollo de los eventos. Recorrer siempre el mismo circuito en los diferentes eventos, ha conducido a una pérdida de interés en los deportistas por participar en los eventos.

Esta investigación resulta relevante ya que puede contribuir para que los hacedores de política deportiva local puedan mejorar la potencialidad turística de los eventos runners. En el trabajo de campo se presentaron dos propuestas, crear un evento "Feria runner Bahía 21k" y crear un circuito que incluya la ciudad, la sierra y el mar, "Circuito ciudad, mar y sierra", aprovechando así las ventajas de localización de la ciudad. Estas propuestas fueron ampliamente aceptadas por los deportistas encuestados y podrían ser un punto de partida para mejorar la poten- cialidad turística de los eventos runners llevados a cabo en la ciudad.

\section{REFERENCIAS}

Alles, M. T. F. (2014). El impacto turístico de los eventos deportivos: un estudio de caso. Cuadernos de turismo, (33), 59-76. Disponible en: https://revistas. um.es/turismo/article/view/195651.

Añó Sanz, V. (2000). La organización de grandes eventos deportivos internacionales. Arbor, $N^{\circ} 165$ (650), pp. 265-287.

Baade, R, Baumann, R y Matheson, V. (2008). Selling the game: Estimating the economic impact of professional sport through taxable sales. Southern Economic Journal. $N^{\circ} 74$ (3), pp, 794-810

Bowerman, W y Harris, W. (1967). "Jogging: A Physical Fitness Program for All Ages". AceBooks.

Brasileiro, M. D. S., Rebollo, S., \& Medina, J. C. C. (2008). Turismo deportivo de litoral: un análisis desde la oferta. Revista Brasileira de Ciências do Esporte, 29(3). Disponible en: http://revista.cbce.org.br/index.php/RBCE/article/view/210.

Brunet, F. (1992). Economía de los Juegos Olímpicos Barcelona'92, Barcelona.

Burgan y Mules. (1992). Economic impact of sporting events. Annals of Tourism Research, № 19 (4), pp, $700-710$

Cerezuela, B. (2003). La información y documentación deportiva y los grandes eventos deportivos.

Crompton J. (2006). "Economic Impact Studies: Instruments for Political Shenanigans?" Journal of Travel Research, vol. 45, pp. 67-82

Crummy, C. (2016). "Conoce al hombre que inventó el aerobic". VICE SPORTS. Disponible en: <https:// sports.vice.com/es/article/kenneth-cooper-inventor-aerobic-ejericio-medicina-fitness/>

Daniels, M.J. (2003): Estimating the economic impact of seven regular sport tourism events. Journal of Sport \& Tourism, vol. 8.4, Enero, pp. 214-222. 
Daniels, M.J.; Norman, W.C. y Henry, M.S. (2004): Estimating effects of a sport tourism event. Annals of Tourism Research, vol. 31.1, Enero, pp. 180-199.

Delpy, L. (2003): An Introduction to Sport and Adventure Tourism. In Sport and Adventure Tourism (S. Hudson, ed.), pp. 1-25, New York: Haworth Hospitality Press.

Gambarota y Leonardi. (2016). Propuesta para transformar un recurso en un atractivo turístico: Centro termal "Termas de La Bahía", Argentina. Revista Interamericana de Ambiente y Turismo. Vol. 12, Núm., 2

González Molina, A. (2008). Rasgos caracterizadores del turismo activo y turismo deportivo e importancia económico-social y estructural de nuevas formas emergentes. Revista Acción Motriz. №1 Julio/Diciembre/2008.

Fixx, J. (1977). The complete book of running. Random House Inc.

Fomento De San Sebastián (2005). Impacto económico del XVI campeonato del mundo de atletismo máster stadia. www.fomentosansebastian.org

Getz, D. (1997). Trends and issues in sport event tourism. Tourism Recreation Research. Vol. 22.2, pp. 61-74.

Getz, D. (1998). Trends, strategies and issues in sport event tourism. Sport Marketing Quartely. Vol. 7 (2), pp. 8-13.
Getz, D. (2003). Sport event tourism: planning, development and marketing. Sport and Adventure Tourism, pp. 49-88

Henderson, J.C.; Foo, K.; Lim, H. Y Yip, S. (2010): Sports events and tourism: the Singapure Formula One Grand Prix. International Journal of Event and Festival Management, vol. 1.3, pp. 60-73

Jiménez Rubio, D. (s/f). Turismo deportivo: las carreras por montaña como herramienta de desarrollo local, Tesis de Magister, Universidad de Alicante. Departamento de Geografía Humana. Disponible en: http://hdl.handle.net/10045/48113.

Kotler, P. (2007): Marketing internacional de lugares y destinos. Pearson. Prentice Hall. México.

Jago, J. y Dwyer, L. (2006). Economic Evaluation of Special Events: a practitioner's guide. Cooperative Research Centre for Sustainable Tourism. Common Ground Publishing Pty Ltd. Australia. En: https://www.researchgate.net/publication/40506787_Economic_Evaluation_of_Special_Events

Latiesa, M. y Paniza, J. (2006). Turistas deportivos: una perspectiva de análisis. Revista Internacional de Sociología (RIS), N 44 (64), pp. 133-149. En: http:// revintsociologia.revistas.csic.es/index.php/revintsociologia/article/viewArticle/31. 
Matheson, V., \& Baade, R. (2004). Mega-sporting events in developing nations: playing the way to prosperity?

Miraglia, D. (2017). El running como práctica turística-recreativa en Bahía Blanca.Potencialidad turística y perfil de la demanda. Tesis de grado inédita. Universidad Nacional de Sur. Departamento de Geografía y Turismo.

Nogueras Carrasco, M. (2010). El deporte y el turismo. Una relación de ahora y para el futuro. En: www.munideporte.com/imagenes/documentacion/ficheros/20100611135518miguel_angel_nogueras.pdf

Ottevanger, H. (2007). Sport Tourism: Factors of influence on sport event visit motivation.

Revuelta, R. (2006). Turismo y eventos deportivos: los Juegos Olímpicos de Invierno. Oppidum. $\mathrm{N}^{\circ} 2$, pp. 399-428.

Sánchez-Fernández, P., Barajas, Á., \& Alén González, M. E. (2013). Los eventos deportivos como herramienta de promoción turística: propuestas para el Rally de Ourense y su entorno (Sport Events as a Tool for Tourism Promotion: Proposals for Rally Ourense and Its Enviroment). Revista de análisis turístico, (16), 59-69.

Santiago, C. (2016). Trail running: modelo e potencial territorial en quanto produto turístico. Dissertação de

Soler Vayá, F., \& San Martín González, E. (2017). La pro- moción del turismo rural a través del trail running: el caso de Carrícola en la comarca de La Valld'Albaida (España). PASOS. Revista de Turismo y Patrimonio Cultural, 15 (1), 49-69.

Solberg, H y Preuss, H. (2007). Major Sport Events and Long-Term Tourism Impacts. Journal of Sport Management. $\mathrm{N}^{\circ} 21$ (2)

Tyrrell, T.J. y Johnston, R.J. (2001): A framework for assessing direct economic impacts of tourist event: distinguishing origins, destinations and causes of expenditures. Journal of Travel Research, vol. 40.1, pp. 94-100.

Tyrrell y Johnson. (2006). The Economic Impacts of Tourism: A Special Issue. Journal of travel Research. Vol45 (1), pp, 3-7

Wilson, R. (2006): "The economic impact of local sport events: significant, limited or otherwise? A case study of 4 swimming events". Managing Leisure, 11(1): 57-70

\section{Páginas Web:}

Actletas (2014). Próceres del running: Bill Bowerman. Disponible en: <http://www.atletas.info/fitness/ proceres-del-running-bill-bowerman/>

EL NUEVO DIARIO. (2009) El libro que cambio el atletismo a nivel mundial. <http://www.elnuevodiario. com.ni/opinion/57104-libro-que-cambio-atletismo-nivel-mundial/> 